\title{
Chen Inequalities for Submanifolds of Real Space Forms with a Ricci Quarter-Symmetric Metric Connection
}

\author{
Nergiz (Önen) Poyraz* Halil İbrahim Yoldaş \\ (Communicated by H. Hilmi Hacısalihoğlu)
}

\begin{abstract}
In this paper, we establish some inequalities for submanifolds of real space forms endowed with a Ricci quarter-symmetric metric connection. Using these inequalities, we obtain the relation between Ricci curvature, scalar curvature and the mean curvature endowed with the Ricci quartersymmetric metric connection.
\end{abstract}

Keywords: Chen inequality; Ricci quarter-symmetric metric connection; Ricci curvature.

AMS Subject Classification (2010): 53B05 ; 53B15; 53C40.

\section{Introduction}

In [8], the idea of a Ricci quarter-symmetric metric connection on a Riemannian manifold was introduced and presented by Kamilya and De. They also found necessary and sufficient conditions for the symmetry of the Ricci tensor of a Ricci quarter-symmetric metric connection and showed that conformal curvature tensor of induced connection $\nabla$ and linear connection $\widetilde{\nabla}$ are equal [8]. Before this work, a few papers had been written about the studies of various types of a quarter-symmetric metric connection and their properties in [11] and [12].

In 1993, Chen [4] introduced a new Riemannian invariant for a Riemannian manifold $M$ as follows:

$$
\delta_{M}=\tau(p)-\inf (K)(p),
$$

where $\tau(p)$ is scalar curvature of $M$ and

$$
\inf (K)(p)=\inf \left\{K(\Pi): K(\Pi) \text { is a plane section of } T_{p} M\right\} .
$$

Chen gave the following general optimal inequality involving the new intrinsic invariant $\delta_{M}$, the squared mean curvature $\|H\|^{2}$ for an $n$-dimensional submanifold $M$ in a real space form $R(c)$ of constant sectional curvature $c:$

$$
\delta_{M} \leq \frac{n^{2}(n-2)}{2(n-2)}\|H\|^{2}+\frac{1}{2}(n+1)(n-2) c .
$$

[3].

Also, Chen established a sharp inequality between the main intrinsic curvatures (the sectional curvature and the scalar curvature) and the main extrinsic curvatures (the squared mean curvature) for a submanifold in real space form $R^{m}(\bar{c})$, well-known as Chen inequalities, in [2] as follows:

For each unit tangent vector $X \in T_{p} M^{n}$,

$$
H^{2}(p) \geq \frac{4}{n^{2}}\{\operatorname{Ric}(X)-(n-1) \bar{c}\},
$$

where $H^{2}$ is the squared mean curvature and $\operatorname{Ric}(X)$ is Ricci curvature of $M^{n}$ at $X$. 
In [7], Hong and Tripathi presented a general inequality for submanifolds of a Riemannian manifold by using (1.3). In [13], this inequality was named Chen-Ricci inequality by Tripathi. In fact, the general inequality obtained in [7] is a special case of Theorem 3.1 of [5]. Later, Mihai and Özgür in [10] proved Chen inequalities for submanifolds of real space forms endowed with a semi-symmetric metric connection. Moreover, several works in this direction is studied $[1,6,9,13]$.

The paper is organized as follows: Section 1 is concerned with introduction. In section 2, we give some basic concepts on submanifolds of Riemannian manifold endowed with Ricci quarter-symmetric metric conection which will be used throughout this paper. In section 3, we find some inequalities for submanifolds of real space forms endowed with a Ricci quarter-symmetric metric connection. Considering these inequalities, we obtain the relation between Ricci curvature, scalar curvature and the mean curvature endowed with the Ricci quarter-symmetric metric connection.

\section{Preliminaries}

Let $\widetilde{M}$ be an $m$-dimensional Riemannian manifold and $\widetilde{\nabla}$ a linear connection on $\widetilde{M}$. A linear connection $\widetilde{\nabla}$ is said to be Ricci quarter-symmetric connection if the torsion tensor $\widetilde{T}$ is of the form

$$
\widetilde{T}(X, Y)=\pi(Y) L X-\pi(X) L Y,
$$

where $\widetilde{\pi}$ is a 1 -form and $L$ is the $(1,1)$ Ricci tensor defined by

$$
\widetilde{g}(L X, Y)=S(X, Y)
$$

$S$ is the Ricci tensor of $\widetilde{M}$.

A linear connection $\widetilde{\nabla}$ is called a metric connection if

$$
\left(\widetilde{\nabla}_{X} \widetilde{g}\right)(Y, Z)=0 .
$$

Following [8], a Ricci quarter-symmetric metric connection $\widetilde{\nabla}$ on $\widetilde{M}$ is given by

$$
\widetilde{\nabla}_{\widetilde{X}} \widetilde{Y}=\stackrel{0}{\nabla}_{\widetilde{X}} \widetilde{Y}+\pi(\widetilde{Y}) L \widetilde{X}-S(\widetilde{X}, \widetilde{Y}) P
$$

for any vector fields $\widetilde{X}$ and $\widetilde{Y}$ of $\widetilde{M}$, where $\stackrel{\circ}{\nabla}$ denotes the Levi-Civita connection with respect to the Riemannian metric $\widetilde{g}, \pi$ is a 1 -form and $P$ is the vector field defined by

$$
\widetilde{g}(P, \widetilde{X})=\pi(\widetilde{X})
$$

for an arbitrary vector field $\widetilde{X}$ of $\widetilde{M}$.

From now on, we will consider a Riemannian manifold $\widetilde{M}$ endowed with a Ricci quarter-symmetric metric connection $\widetilde{\nabla}$ and the Levi-Civita connection denoted by $\stackrel{\circ}{\widetilde{\nabla}}$.

Let $M^{n}$ be an $n$-dimensional submanifold of an $m$-dimensional Riemannian manifold $\widetilde{M}$. On the submanifold $M^{n}$ we consider the induced Ricci quarter-symmetric metric connection denoted by $\nabla$ and the induced Levi-Civita connection denoted by $\stackrel{\circ}{\nabla}$.

Let $\widetilde{R}$ be the curvature tensor of $\widetilde{M}$ with respect to $\widetilde{\nabla}$ and $\stackrel{\circ}{\widetilde{R}}$ the curvature tensor of $\widetilde{M}$ with respect to $\stackrel{\circ}{\widetilde{\nabla}}$. We also denote by $R$ and $\stackrel{\circ}{R}$ the curvature tensors of $\nabla$ and $\stackrel{\circ}{\nabla}$, respectively, on $M$.

The Gauss formulas with respect to $\nabla$ and $\stackrel{\circ}{\nabla}$, respectively, can be written as:

$$
\begin{aligned}
& \widetilde{\nabla}_{X} Y=\nabla_{X} Y+h(X, Y), \\
& \stackrel{\circ}{\nabla}_{X} Y=\stackrel{\circ}{\nabla}_{X} Y+\stackrel{\circ}{h}(X, Y),
\end{aligned}
$$

where $\stackrel{\circ}{h}$ is the second fundemental form of $M$ in $\widetilde{M}$ and $h$ is a (0,2)-tensor on $M$. 
For any orthonormal basis $\left\{e_{1}, \ldots, e_{n}\right\}$ of the tangent space $T_{p} M^{n}$, the mean curvature vector $H(p)$ is given by

$$
H(p)=\frac{1}{n} \sum_{i=1}^{n} h\left(e_{i}, e_{i}\right)
$$

If $h=0$ ( respectively $H=0$ ), then the submanifold $M^{n}$ is called totally geodesic (minimal) in $\widetilde{M}$. If $h(X, Y)=$ $g(X, Y) H$ for all $X, Y \in T M$, then $M^{n}$ is said to be totally umbilical.

The Gauss equation with respect to the Ricci quarter-symmetric metric connection is

$$
R(X, Y, Z, W)=\tilde{R}(X, Y, Z, W)+g(h(X, Z), h(Y, W))-g(h(X, W), h(Y, Z)) .
$$

The curvature tensor $\stackrel{\circ}{\widetilde{R}}$ with respect to the Levi-Civita connection $\stackrel{\circ}{\widetilde{\nabla}}$ on $\widetilde{M}(c)$ is expressed by

$$
\stackrel{\circ}{\widetilde{R}}(X, Y, Z, W)=c\{g(X, W) g(Y, Z)-g(Y, W) g(X, Z)\} .
$$

Let $\Pi=\operatorname{Span}\left\{e i_{i}, e_{j}\right\}$ be 2 -dimensional non-degenerate plane of the tangent space $T p M$ at $p \in M$. Then the number

$$
K_{i j}=\frac{g\left(R\left(e_{j}, e_{i}\right) e_{i}, e_{j}\right)}{g\left(e_{i}, e_{i}\right) g\left(e_{j}, e_{j}\right)-g\left(e_{i}, e_{j}\right)^{2}}
$$

is called the sectional curvature of the section $\Pi$ at $p \in M$.

Let $M^{n}$ be an $n$-dimensional Riemanian manifold. We denote by $K(\pi)$ the sectional curvature of $M^{n}$ associated with a plane section $\pi \subset T p M^{n}, p \in M^{n}$. If $\left\{e_{1}, \ldots, e_{n}\right\}$ is an orthonormal basis of the tangent space $T p M^{n}$, then the scalar curvature $\tau$ at $p$ is defined by

$$
\tau(p)=\sum_{1 \leq i<j \leq n} K_{i j}
$$

Let $M^{n}$ be an $n$-dimensional Riemannian manifold, $L$ be a $k$-plane section of $T p M^{n}, p \in M^{n}$, and $X$ be a unit vector in $L$. We choose an orthonormal basis $\left\{e_{1}, \ldots, e_{k}\right\}$ of $\mathrm{L}$ such that $e_{1}=X$.

One defines [2] the Ricci curvature (or $k$-Ricci curvature) of $L$ at $X$ by

$$
\operatorname{Ric}_{L}(X)=K_{12}+K_{13}+\ldots+K_{1 k},
$$

where $K_{i j}$ denotes, as usual, the sectional curvature of the 2-plane section spanned by $e_{i}, e_{j}$. For each integer $k, 2 \leq k \leq n$, the Riemannian invariant $\theta_{k}$ on $M^{n}$ is defined by:

$$
\theta_{k}(p)=\frac{1}{k-1} \inf _{L, X} \operatorname{Ric}_{L}(X), \quad p \in M,
$$

where $L$ runs over all $k$-plane sections in $T p M^{n}$ and $X$ runs over all unit vectors in $L$.

Then the curvature tensor $\widetilde{R}$ with respect to the Ricci quarter-symmetric metric connection $\widetilde{\nabla}$ on $\widetilde{M}$ can be shown that $[8]$

$$
\begin{aligned}
\widetilde{R}(X, Y) Z= & \stackrel{\circ}{\widetilde{R}}(X, Y) Z-M(Y, Z) L X+M(X, Z) L Y \\
& -S(Y, Z) Q X+S(X, Z) Q Y+\pi(Z)\left[\left(\stackrel{\circ}{\nabla}_{X} L\right) Y-\left(\stackrel{\circ}{\nabla}_{Y} L\right) X\right] \\
& -\left[\left(\stackrel{\circ}{\nabla}_{X} S\right)(Y, Z)-\left(\stackrel{\circ}{\nabla}_{Y} S\right)(X, Z)\right] P,
\end{aligned}
$$

where $M$ is tensor field of type $(0,2)$ defined by

$$
M(X, Y)=g(Q X, Y)=\left(\stackrel{\circ}{\nabla}_{X} \pi\right) Y-\pi(Y) \pi(L X)+\frac{1}{2} \pi(P) S(X, Y)
$$

and $Q$ is a tensor field of type $(2,1)$ defined by

$$
Q X=\stackrel{\circ}{\nabla}_{X} P-\pi(L X) P+\frac{1}{2} \pi(P) L X
$$


Here we shall consider $M^{n}$ to be an Einstein manifold, that is,

$$
S(X, Y)=\stackrel{\stackrel{\circ}{\tau}}{n} g(X, Y)
$$

where $\stackrel{0}{\widetilde{\tau}}$ is the scalar curvature.

Throughout this paper, we assume that $M^{n}$ is an Einstein manifold.

Considering (2.11) and (2.14) we get

$$
\widetilde{R}(X, Y) Z=\stackrel{\circ}{\widetilde{R}}(X, Y) Z-\frac{\stackrel{\circ}{\widetilde{\tau}}}{n}[M(Y, Z) X-M(X, Z) Y+g(Y, Z) Q X-g(X, Z) Q Y] .
$$

Contracting (2.15) with respect to $X$, we get

$$
\widetilde{S}(Y, Z)=\frac{\stackrel{\circ}{\widetilde{\tau}}}{n}[g(Y, Z)-\{(n-2) M(Y, Z)+m g(Y, Z)\}],
$$

where $\widetilde{S}$ is the Ricci tensor of $\widetilde{\nabla}$ and $m$ is the trace of $M(Y, Z)$. Now putting $Y=Z=e_{i}$, where $\left\{e_{1}, \ldots, e_{n}\right\}$ is an orthonormal basis of the tangent space at any point, we get by taking the sum for $1 \leq i \leq n$ in the relation (2.16)

$$
\widetilde{\tau}=\frac{\stackrel{\circ}{\tau}}{n}[n-2(n-1) m],
$$

where $\widetilde{\tau}$ is the scalar curvature of $\widetilde{\nabla}$.

\section{3. k-Ricci Curvature and k-Scalar Curvature}

In this section, a sharp relation between the Ricci curvature in the direction of unit tangent vector $X$ and the mean curvature $H$ with respect to Ricci quarter-symmetric metric connection $\widetilde{\nabla}$ is established. Using this inequality, a relationship between the $k$-Ricci curvature of $M^{n}$ and the squared mean curvature $\|H\|^{2}$ is showed. From now on, we assume that the vector field $P$ is tangent to $M^{n}$.

Denote by

$$
N(p)=\left\{X \in T_{p} M^{n} \mid h(X, Y)=0, \forall Y \in T_{p} M^{n}\right\} .
$$

Theorem 3.1. Let $M^{n}$ be an $n$-dimensional submanifold of an $m$-dimensional real space form $\widetilde{M}(c)$ of constant sectional curvature $c$ endowed with Ricci quarter-symmetric metric connection $\widetilde{\nabla}$. Then, the following statements are true.

(i) For each unit vector $X \in T_{p} M^{n}$ we have

$$
\operatorname{Ric}(X) \leq \frac{1}{4} n^{2}\|H\|^{2}-(n-1) c[1-m+(n-2) M(X, X)],
$$

where $m$ is the trace of $M$.

(ii) The equality case of (3.1) is satisfied by unit vector $X \in T_{p} M^{n}$ if and only if

$$
\begin{aligned}
h(X, Y) & =0, \text { for all } Y \in T p M^{n} \text { orthogonal to } X, \\
h(X, X) & =\frac{n}{2} H(p) .
\end{aligned}
$$

(iii) The equality case of (3.1) holds for all unit vector $X \in T_{p} M^{n}$ if and only if either $p$ is a totally geodesic point or $n=2$ and $p$ is a totally umbilical point.

Proof. From (2.7) and (2.15) we get

$$
2 \tau(p)=n(n-1) c-2(n-1)^{2} c m+n^{2}\|H\|^{2}-\|h\|^{2},
$$


where $m$ is the trace of $M$ and denote by

$$
\|h\|^{2}=\sum_{i, j=1}^{n} g\left(h\left(e_{i}, e_{j}\right), h\left(e_{i}, e_{j}\right)\right) .
$$

From (3.3), we get

$$
\begin{aligned}
\frac{1}{4} n^{2}\|H\|^{2}= & \tau(p)-\frac{n(n-1) c}{2}+(n-1)^{2} c m \\
& +\frac{1}{4} \sum_{r=n+1}^{m}\left(h_{11}^{r}-h_{22}^{r}-\ldots-h_{n n}^{r}\right)^{2}+\sum_{r=n+1}^{m} \sum_{j=2}^{n}\left(h_{1 j}^{r}\right)^{2} \\
& -\sum_{r=n+1}^{m} \sum_{2 \leq i<j \leq n}^{m}\left(h_{i i}^{r} h_{j j}^{r}-\left(h_{i j}^{r}\right)^{2}\right),
\end{aligned}
$$

where

$$
h_{i j}^{r}=g\left(h\left(e_{i}, e_{j}\right), e_{r}\right)
$$

Using (2.7) and (2.15) we also have

$$
\begin{aligned}
\sum_{r=n+1}^{m} \sum_{2 \leq i<j \leq n}^{m}\left(h_{i i}^{r} h_{j j}^{r}-\left(h_{i j}^{r}\right)^{2}\right) & =\sum_{2 \leq i<j \leq n} K_{i j}-\sum_{2 \leq i<j \leq n} \tilde{K}_{i j} \\
& =\sum_{2 \leq i<j \leq n} K_{i j}-(n-1)(n-2) c\left(\frac{1}{2}-m+M\left(e_{1}, e_{1}\right)\right) .
\end{aligned}
$$

From (3.4) and (3.5), we obtain

$$
\begin{aligned}
\operatorname{Ric}\left(e_{1}\right)= & \frac{1}{4} n^{2}\|H\|^{2}-(n-1) c\left(1-m+(n-2) M\left(e_{1}, e_{1}\right)\right) \\
& -\sum_{r=n+1}^{m} \sum_{j=2}^{n}\left(h_{1 j}^{r}\right)^{2}-\frac{1}{4} \sum_{r=n+1}^{m}\left(h_{11}^{r}-h_{22}^{r}-\ldots-h_{n n}^{r}\right)^{2} .
\end{aligned}
$$

If we choose $e_{1}=X$ as any unit vector of $T_{p} M^{n}$ in the above equation, one obtains (3.1).

Taking into consideration equation (3.6) and $X=e_{1}$, the equality case of (3.1) holds if and only if

$$
h_{12}^{r}=h_{13}^{r}=\ldots=h_{1 n}^{r}=0 \text { and } h_{11}^{r}=h_{22}^{r}+\ldots+h_{n n}^{r}, r \in\{n+1, \ldots, m\}
$$

which shows that (3.2) holds.

We now suppose that the equality case of (3.1) holds for all unit vector $X \in T_{p} M^{n}$. Then , in view of (3.7), for each $r \in\{n+1, \ldots, m\}$ we have $i \in\{1, \ldots, n\}$,

$$
\begin{aligned}
h_{i j}^{r} & =0, \quad i \neq j \\
2 h_{i i}^{r} & =h_{11}^{r}+h_{22}^{r}+\ldots+h_{n n}^{r}, \quad i \in\{1, \ldots, n\} .
\end{aligned}
$$

From (3.9), we have $2 h_{11}^{r}=2 h_{22}^{r}=\ldots=2 h_{n n}^{r}=h_{11}^{r}+h_{22}^{r}+\ldots+h_{n n}^{r}$, which implies that

$$
(n-2)\left(h_{11}^{r}+h_{22}^{r}+\ldots+h_{n n}^{r}\right)=0 \text {. }
$$

Thus, either $h_{11}^{r}+h_{22}^{r}+\ldots+h_{n n}^{r}=0$ or $n=2$. If $h_{11}^{r}+h_{22}^{r}+\ldots+h_{n n}^{r}=0$, then in view of (3.9), we get $h_{i i}^{r}=0$ for all $i \in\{1, \ldots, n\}$. This together with (3.8) gives $h_{i j}^{r}=0$ for all $i, j \in\{1, \ldots, n\}$ and $r \in\{n+1, \ldots, m\}$, that is, $p$ is a totally geodesic point. If $n=2$, then from (3.9) $2 h_{11}^{r}=2 h_{22}^{r}=h_{11}^{r}+h_{22}^{r}$, which shows that $p$ is a totally umbilical point. The proof of the converse part is straightforward.

Corollary 3.1. If $H(p)=0$, then a unit tangent vector $X$ at $p$ satisfies the equality case of (3.1) if and only if $X \in N(p)$.

Theorem 3.2. Let $M^{n}$ be an $n$-dimensional submanifold of an $m$-dimensional real space form $\widetilde{M}(c)$ of constant sectional curvature $c$ endowed with Ricci quarter-symmetric metric connection $\widetilde{\nabla}$

$$
\tau(p) \leq \frac{(n-1)}{2}\left(n\|H\|^{2}+n c-2 c(n-1) m\right) .
$$

Equality case of (3.11) holds at $p \in M^{n}$ if and only if $p$ is a totally umbilical point. 
Proof. Let $p \in M^{n}$ and $\left\{e_{1}, \ldots, e_{n}\right\}$ be orthonormal basis of $T_{p} M^{n}$. The relation (3.3) is equivalent to

$$
n^{2}\|H\|^{2}=2 \tau(p)+\|h\|^{2}+(n-1) c(2(n-1) m-n) .
$$

We choose an orthonormal basis $\left\{e_{1}, \ldots, e_{n}, e_{n+1}, \ldots, e_{m}\right\}$ at $\mathrm{p}$ such that $e_{n+1}$ is parallel to the mean curvature vector $H(p)$ and $e_{1}, \ldots, e_{n}$ diagonalize the shape operator $A_{e_{n+1}}$. Then the shape operators take the forms

$$
\begin{gathered}
A_{e_{n+1}}=\left[\begin{array}{cccccc}
a_{1} & 0 & . & . & . & 0 \\
0 & a_{2} & \cdot & \cdot & . & 0 \\
\cdot & \cdot & \cdot & & . \\
\cdot & \cdot & & \cdot & . \\
\cdot & \cdot & & . & \cdot \\
0 & 0 & . & . & \cdot & a_{n}
\end{array}\right] \\
A_{e_{r}}=\left(h_{i j}^{r}\right), \quad i, j=1, \ldots, n ; \quad r=n+2, \ldots, m, \quad \text { trace } A_{e_{r}}=0 .
\end{gathered}
$$

From (3.12), we get

$$
n^{2}\|H\|^{2}=2 \tau(p)+\sum_{i=1}^{n} a_{i}^{2}+\sum_{r=n+2}^{m} \sum_{i, j=1}^{n}\left(h_{i j}^{r}\right)^{2}+(n-1) c(2(n-1) m-n) .
$$

On the other hand, since

$$
0 \leq \sum_{i<j}\left(a_{i}-a_{j}\right)^{2}=(n-1) \sum_{i} a_{i}^{2}-2 \sum_{i<j} a_{i} a_{j}
$$

we obtain

$$
n^{2}\|H\|^{2}=\left(\sum_{i=1}^{n} a_{i}\right)^{2}=\sum_{i=1}^{n} a_{i}^{2}+2 \sum_{i<j} a_{i} a_{j} \leq n \sum_{i=1}^{n} a_{i}^{2}
$$

which implies

$$
\sum_{i=1}^{n} a_{i}^{2} \geq n\|H\|^{2}
$$

So from (3.15) and (3.18), we have

$$
n^{2}\|H\|^{2} \geq 2 \tau(p)+n\|H\|^{2}+\sum_{r=n+2}^{m} \sum_{i, j=1}^{n}\left(h_{i j}^{r}\right)^{2}+(n-1) c(2(n-1) m-n) .
$$

If the equality case of (3.11) holds, then from (3.16) and (3.19) it follows that

$$
a_{1}=a_{2}=\ldots=a_{n} \text { and } A_{e_{r}}=0, r=n+2, \ldots, m .
$$

Therefore, $p$ is a totally umbilical point. The converse is straightforward.

Theorem 3.3. Let $M^{n}$ be an $n$-dimensional submanifold of an $m$-dimensional real space form $\widetilde{M}(c)$ of constant sectional curvature $c$ endowed with Ricci quarter-symmetric metric connection $\widetilde{\nabla}$. Then we have

$$
\theta_{k}(p) \leq\|H\|^{2}+c\left(2-\frac{4(n-1) m}{n}\right) .
$$

Proof. Let $\left\{e_{1}, \ldots, e_{n}\right\}$ be an orthonormal basis of $T_{p} M^{n}$. Denote by $L_{i_{1} \ldots i_{k}}$ the $k$-plane section spanned by $\left\{e_{i_{1}}, \ldots, e_{i_{k}}\right\}$. Using the definitions of the ricci and scalar curvatures, we have

$$
\begin{gathered}
\tau\left(L_{i_{1} \ldots i_{k}}\right)=\frac{1}{2} \sum_{i \in\left\{i_{1}, \ldots, i_{k}\right\}} \operatorname{Ric}_{L_{i_{1} \ldots i_{k}}}\left(e_{i}\right), \\
\tau(p)=\frac{1}{C_{n-2}^{k-2}} \sum_{1 \leq i_{1}<\ldots \leq i_{k} \leq n} \tau\left(L_{i_{1} \ldots i_{k}}\right) .
\end{gathered}
$$

From (2.10), (3.22) and (3.23), we get

$$
\tau(p) \geq \frac{n(n-1)}{2} \theta_{k}(p)
$$

Using (3.11) and (3.24) we obtain (3.21). 
Lemma 3.1. If $n>k \geq 2$ and $a_{1}, \ldots, a_{n}$, a are real numbers such that

$$
\left(\sum_{i=1}^{n} a_{i}\right)^{2}=(n-k+1)\left(\sum_{i=1}^{n} a_{i}^{2}+a\right)
$$

then

$$
2 \sum_{1 \leq i<j \leq k} a_{i} a_{j} \geq a
$$

with equality holding if and only if

$$
a_{1}+a_{2}+\ldots+a_{k}=a_{k+1}=\ldots=a_{n} .
$$

Theorem 3.4. Let $M^{n}$ be an $n$-dimensional submanifold of an $m$-dimensional real space form $\widetilde{M}(c)$ of constant sectional curvature $c$ endowed with Ricci quarter-symmetric metric connection $\widetilde{\nabla}$. Then, for each point $p \in M^{n}$ and each $k$-plane section $\Pi_{k} \subset T p M^{n}(n>k \geq 2)$, we have

$$
\begin{aligned}
\tau(p)-\tau\left(\pi_{k}\right) \leq & (n-k)\left[\frac{n^{2}}{2(n-k+1)}\|H\|^{2}-\frac{(n+k-1)}{2} c-(n-1) c m\right] \\
& -(n-1)(k-1) \operatorname{ctrace}\left(m_{\left.\right|_{\pi_{\frac{1}{k}}}}\right) .
\end{aligned}
$$

The equality case of (3.28) holds at $p \in M^{n}$ if and only if there exist an orthonormal basis $\left\{e_{1}, \ldots, e_{n}\right\}$ of $T p M^{n}$ and an orthonormal basis $\left\{e_{n+1}, \ldots, e_{m}\right\}$ of $T_{p}^{\perp} M^{n}$ such that $(a) \Pi_{k}=\operatorname{Span}\left\{e_{1}, \ldots, e_{k}\right\}$ and (b) the forms of shape operators $A_{e_{r}}$, $r=n+1, \ldots, m$, take the forms

$$
\begin{aligned}
& A_{e_{n+1}}=\left[\begin{array}{ccccccc}
h_{11}^{n+1} & 0 & \cdot & \cdot & \cdot & 0 & \\
0 & h_{22}^{n+1} & \cdot & \cdot & \cdot & 0 & 0 \\
\cdot & \cdot & \cdot & & & \\
\cdot & \cdot & & \cdot & & & \\
\cdot & \cdot & & \cdot & \cdot & h_{k k}^{n+1} & \\
0 & 0 & \cdot & \cdot & \cdot & \left.h_{k k}^{k} h_{i i}^{n+1}\right) I_{n-k}
\end{array}\right] \\
& A_{e_{r}}=\left[\begin{array}{ccccccc}
h_{11}^{r} & h_{12}^{r} & \cdot & \cdot & \cdot & h_{1 k}^{r} & \\
h_{12}^{r} & h_{22}^{r} & \cdot & \cdot & \cdot & h_{2 k}^{r} & 0 \\
\cdot & \cdot & \cdot & & & \\
\cdot & \cdot & & \cdot & & & \\
\cdot & \cdot & & & \cdot & & \\
h_{1 k}^{r} & h_{2 k}^{r} & \cdot & \cdot & \cdot & -\sum_{i=1}^{k-1} h_{i i}^{r} & \\
& & 0 & & & 0_{n-k}
\end{array}\right], \quad r \in\{n+2, \ldots, m\} .
\end{aligned}
$$

Proof. Let $\Pi_{k} \subset T p M^{n}$ be a $k$-plane section. We choose an orthonormal basis $\left\{e_{1}, \ldots, e_{n}\right\}$ for $T p M^{n}$ and $\left\{e_{n+1}, \ldots, e_{m}\right\}$ for the normal space $T_{p}^{\perp} M^{n}$ at $p$ such that $\Pi_{k}=\operatorname{Span}\left\{e_{1}, \ldots, e_{k}\right\}$, the mean curvature vector $H$ is in the direction of the normal vector to $e_{n+1}$ and $e_{1}, \ldots, e_{n}$ diagonalize the shape operator $A_{e_{n+1}}$. Then the shape operators take the forms (3.13) and (3.14). We rewrite (3.3) as

$$
\left(\sum_{i=1}^{n} h_{i i}^{n+1}\right)^{2}=(n-k+1)\left(\sum_{i=1}^{n}\left(h_{i i}^{n+1}\right)^{2}+\sum_{r=n+2}^{m} \sum_{i, j=1}^{n}\left(h_{i j}^{r}\right)^{2}+\epsilon\right),
$$

where

$$
\epsilon=2 \tau(p)-n(n-1) c+2(n-1)^{2} c m-\frac{n^{2}(n-k)}{(n-k+1)}\|H\|^{2} .
$$

Applying Lemma 3.1 in (3.31), we get

$$
2 \sum_{1 \leq i<j \leq k} h_{i i}^{n+1} h_{j j}^{n+1} \geq \epsilon+\sum_{r=n+2}^{m} \sum_{i, j=1}^{n}\left(h_{i j}^{r}\right)^{2} .
$$


From equation (2.7) and (2.15) it also follows that

$$
\begin{aligned}
\tau\left(\pi_{k}\right)= & \frac{k(k-1) c}{2}-(n-1)(k-1) c \sum_{i=1}^{k} M\left(e_{i}, e_{i}\right)+\sum_{1 \leq i<j \leq k} h_{i i}^{n+1} h_{j j}^{n+1} \\
& +\sum_{r=n+2}^{m} \sum_{1 \leq i<j \leq k}^{m}\left(h_{i i}^{r} h_{j j}^{r}-\left(h_{i j}^{r}\right)^{2}\right) .
\end{aligned}
$$

Using (3.33) and (3.34) we get

$$
\begin{aligned}
\tau\left(\pi_{k}\right) \geq & \frac{k(k-1) c}{2}-(n-1)(k-1) c \sum_{i=1}^{k} M\left(e_{i}, e_{i}\right)+\frac{1}{2} \epsilon \\
& +\frac{1}{2} \sum_{r=n+2}^{m}\left(h_{11}^{r}+h_{22}^{r}+\ldots+h_{k k}^{r}\right)^{2}+\frac{1}{2} \sum_{r=n+2}^{m} \sum_{i, j>k}^{n}\left(h_{i j}^{r}\right)^{2} \\
& +\sum_{r=n+2}^{m} \sum_{j>k}^{n}\left(\left(h_{1 j}^{r}\right)^{2}+\left(h_{2 j}^{r}\right)^{2}+\ldots+\left(h_{k j}^{r}\right)^{2}\right)
\end{aligned}
$$

or

$$
\tau\left(\pi_{k}\right) \geq \frac{k(k-1) c}{2}-(n-1)(k-1) c \sum_{i=1}^{k} M\left(e_{i}, e_{i}\right)+\frac{1}{2} \epsilon .
$$

We remark that

$$
M\left(e_{1}, e_{1}\right)+M\left(e_{2}, e_{2}\right)+\ldots+M\left(e_{k}, e_{k}\right)=m-\operatorname{trace}\left(m_{\left.\right|_{\pi_{k}}}\right) .
$$

From (3.32), (3.36) and (3.37) we obtain

$$
\begin{aligned}
\tau\left(\pi_{k}\right) \geq & (n-k)\left(\frac{(n+k-1)}{2} c+(n-1) c m\right)+\tau(p) \\
& -\frac{n^{2}(n-k)}{2(n-k+1)}\|H\|^{2}+(n-1)(k-1) \operatorname{ctrace}\left(m_{\left.\right|_{\pi_{k}}}\right)
\end{aligned}
$$

which proves the inequality case of (3.28).

If the equality case of (3.28) holds, then the inequalities given by (3.33) and (3.36) become equalities. In this case, for $r=n+2, \ldots, m$ we have

$$
\begin{gathered}
h_{1 j}^{n+1}=h_{2 j}^{n+1}=\ldots=h_{k j}^{n+1}=0, \quad j=k+1, \ldots, n, \\
h_{i j}^{r}=0, \quad i, j=k+1, \ldots, n, \\
h_{11}^{r}+h_{22}^{r}+\ldots+h_{k k}^{r}=0 .
\end{gathered}
$$

Applying Lemma 3.1 we also have

$$
h_{11}^{n+1}+h_{22}^{n+1}+\ldots+h_{k k}^{n+1}=h_{l l}^{n+1}, \quad l=k+1, \ldots, n .
$$

Thus, after choosing a suitable orthonormal basis $\left\{e_{1}, \ldots, e_{m}\right\}$, the shape operator of $M^{n}$ takes the form given by (3.29) and (3.30). The converse is easy to follow.

By Theorem 3.4 we get the following corollary.

Corollary 3.2. Let $M^{n} n \geq 3$, be an $n$-dimensional submanifold of an $m$-dimensional real space form $\widetilde{M}(c)$ of constant sectional curvature $c$ endowed with Ricci quarter-symmetric metric connection $\widetilde{\nabla}$. Then, for each point $p \in M^{n}$ and each 2-plane section $\Pi_{2} \subset T p M^{n}$, we have

$$
\begin{aligned}
\delta_{M} \leq & (n-2)\left[\frac{n^{2}}{2(n-1)}\|H\|^{2}-\frac{(n+1)}{2} c-(n-1) c m\right] \\
& -(n-1) \operatorname{ctrace}\left(m_{\left.\right|_{\pi^{\perp}}}\right) .
\end{aligned}
$$


The equality case of (3.43) holds at $p \in M^{n}$ if and only if there exist an orthonormal basis $\left\{e_{1}, \ldots, e_{2}\right\}$ of $T p M^{n}$ and an orthonormal basis $\left\{e_{n+1}, \ldots, e_{m}\right\}$ of $T_{p}^{\perp} M^{n}$ such that $(a) \Pi_{2}=\operatorname{Span}\left\{e_{1}, e_{2}\right\}$ and $(b)$ the forms of shape operators $A_{e_{r}}$, $r=n+1, \ldots, m$, become

$$
A_{e_{n+1}}=\left[\begin{array}{ccc}
a & 0 & 0 \\
0 & b & 0 \\
0 & 0 & (a+b) I_{n-2}
\end{array}\right], A_{e_{r}}=\left[\begin{array}{ccc}
c_{r} & d_{r} & 0 \\
d_{r} & -c_{r} & 0 \\
0 & 0 & 0_{n-2}
\end{array}\right], r=n+2, \ldots, m
$$

\section{References}

[1] Chen, B. Y., Mean curvature and shape operator of isometric immersion in real space forms. Glasgow Mathematic Journal 38 (1996), $87-97$.

[2] Chen, B. Y., Relation between Ricci curvature and shape operator for submanifolds with arbitrary codimension. Glasgow Mathematic Journal 41 (1999), 33-41.

[3] Chen, B. Y., Some pinching and classification theorems for minimal submanifolds. Arch. math (Basel) 60 (1993), no. 6, 568-578.

[4] Chen, B. Y., A Riemannian invariant for submanifolds in space forms and its applications. Geometry and Topology of submanifolds VI. (Leuven, 1993/Brussels, 193). (NJ:Word Scientific Publishing, River Edge). 1994, pp. 58-81, no. 6, 568-578.

[5] Chen, B. Y., A general optimal inequlaity for arbitrary Riemannian submanifolds. J. Ineq. Pure Appl. Math 6 (2005), no. 3, Article 77, 1-11.

[6] Gülbahar, M., Kılıç, E., Keleş, S. and Tripathi, M. M., Some basic inequalities for submanifolds of nearly quasi-constant curvature manifolds. Differential Geometry-Dynamical Systems. 16 (2014), 156-167.

[7] Hong, S. and Tripathi, M. M., On Ricci curvature of submanifolds. Int J. Pure Appl. Math. Sci. 2 (2005), no.2, 227-245.

[8] Kamilya, D and De, U. C., Some properties of a Ricci quarter-symmetric metric connection in a Riemanian manifold. Indian J. Pure and Appl. Math 26 (1995), no. 1, 29-34.

[9] Liu, X. and Zhou, J., On Ricci curvature of certain submanifolds in cosympletic space form. Sarajeva J. Math 2 (2006), no.1, 95-106.

[10] Mihai, A. and Özgür, C., Chen inequalities for submanifolds of real space form with a semi-symmetric metric connection. Taiwanese Journal of Mathematics 14 (2010), no. 4, 1465-1477.

[11] Mishra, R. S. and Pandey, S. N., On quarter symmetric metric F-connections. Tensor (N.S.) 34 (1980), no. 1, 1-7.

[12] Rastogi, S. C., On quarter-symmetric metric connection. C. R. Acad. Bulgare Sci 31 (1978), no. 7, 811-814.

[13] Tripathi, M. M., Improved Chen-Ricci inequality for curvature-like tensor and its applications. Differential Geom. Appl. 29 (2011), 685-698.

\section{Affiliations}

\section{NERGIZ (ÖNEN) POYRAZ}

ADDRESS: Cukurova University, Department of Mathematics, 01330, Adana-Turkey.

E-MAIL: nonen@cu.edu.tr

ORCID ID: orcid.org/0000-0002-8110-712X

HALIL İBRAHIM YOLDAŞ

ADDRESS: Mersin University, Departmet of Mathematics, 33343, Mersin-Turkey.

E-MAIL: hibrahimyoldas@mersin.edu.tr

ORCID ID: orcid.org/0000-0002-3238-6484 